\title{
Comment on: Estimate of incidence of ROP requiring treatment in extreme preterms and impact on service -7 year review in tertiary unit
}

\author{
Jane R. MacKinnon ${ }^{1} \cdot$ Liying Low $^{1} \cdot$ Rishi Sharma ${ }^{1} \cdot$ James Yi Lim $^{1}$ \\ Received: 11 June 2019 / Accepted: 18 September 2019 / Published online: 4 October 2019 \\ (c) The Royal College of Ophthalmologists 2019
}

\section{To the Editor:}

We thank Tavassoli et al. for their report on the increase in number of neonates requiring laser treatment for retinopathy of prematurity (ROP) seen in the UK associated with the improved survival of extremely preterm babies [1]. In addition, we wish to draw attention to those preterm infants at higher gestational ages with congenital abnormalities who previously had significant mortality, but who now survive the neonatal period, and who are also at risk of ROP. We illustrate this with a case of genetically confirmed Beckwith-Wiedemann syndrome (BWS) in a Caucasian neonate born at $30+5$ weeks, birth weight $3360 \mathrm{~g}$, who developed unexpectedly severe ROP.

During ROP screening she developed two distinct ridges: the anterior being avascular while the more posterior ridge contained a proliferation of vessels over several clock hours in both eyes with plus disease. At a corrected gestational age of 40 weeks she underwent bilateral diode laser to the avascular retina under GA, which was effective in preventing retinal detachment.

This infant had the typical clinical features of BWS (macroglossia, macrosomia, neonatal hypoglycaemia, abnormal ear pits/creases and midline abdominal wall defect) but in addition had a pancreatic tumour resulting in confirmed hyperinsulinaemia with associated hypoglycaemia. Following near-total pancreatectomy a tapering dose of insulin was required over a 2 -week period.

This large-for-gestational-age infant was not expected to develop significant ROP based on her maturity, however the

Jane R. MacKinnon

Jane.mackinnon1@nhs.net

1 Ophthalmology Department, Royal Hospital for Sick Children, Glasgow G3 8SJ, Scotland presence of BWS causing abnormal glucose homeostasis necessitated significant medical and surgical intervention for survival.

In 1986 the mortality rate for neonates with BWS was reported to be $20 \%$ [2], however details from a panEuropean study published in 2018 suggest that this rate has approximately halved [3].

Advances in neonatal care for preterm infants are welcome. Recognition of the impact this has on an ophthalmologist's workload should be highlighted as in Tavassoli et al.'s paper. Improved survival of premature neonates with congenital abnormalities as well as extreme preterm infants inevitably results in an increased burden of screening and treatment for ROP.

\section{Compliance with ethical standards}

Conflict of interest The authors declare they have no conflict of interest.

Publisher's note Springer Nature remains neutral with regard to jurisdictional claims in published maps and institutional affiliations.

\section{References}

1. Tavassoli S, Wach R, Haynes R, Markham R, Williams C. Estimate of incidence of ROP requiring treatment in extreme preterms and impact on service -7 year review in tertiary unit. Eye. 2019;33:845-9.

2. Pettenati MJ, Haines JL, Higgins RR, Wappner RS, Palmer CG, Weaver DD. Wiedemann-Beckwith syndrome: presentation of clinical and cytogenetic data on 22 new cases and review of the literature. Hum Genet. 1986;74:143-54.

3. Barisic I, Boban L, Akhmedzhanova D, Bergman JEH, CaveroCarbonell C, Grinfelde I, et al. Beckwith Wiedemann syndrome: a population-based study on prevalence, prenatal diagnosis, associated anomalies and survival in Europe. Eur $\mathrm{J}$ Med Genet. 2018;61:499-507. 